\title{
Conocimiento del Profesorado de Infantil y Primaria en Reanimación Cardiopulmonar Knowledge of Cardiopulmonary Resuscitation in Kindergarten and Primary School Teachers
}

\author{
Zuriñe Gaintza, Zaloa Velasco
}

Universidad del País Vasco (España), Servicio de Salud Vasco (España)

\begin{abstract}
Resumen. En España, la legislación educativa (LOMCE, 2013) incluye la necesidad de formar al alumnado en Primeros Auxilios y la investigación señala la importancia de conocer las técnicas de Reanimación Cardiopulmonar (RCP). En este contexto, con el objetivo de evaluar el conocimiento del profesorado de infantil y primaria $(\mathrm{N}=361)$ en $\mathrm{RCP}$ se realiza un estudio descriptivo aplicando un cuestionario dividido en dos partes: datos demográficos y preguntas relacionadas con el conocimiento teórico en primeros auxilios. Los resultados referentes a RCP señalan que el profesorado no tiene conocimientos, no existiendo diferencias significativas en relación con la edad, el género, con los estudios realizados ni con el tiempo de trabajo ( $p>0,05)$. Sólo existen diferencias significativas si se ha realizado un curso en primeros auxilios o no. En base a los resultados se concluye que es necesario formar en primeros auxilios poniendo especial atención en la reanimación cardiopulmonar tanto al alumnado de los grados de educación infantil y primaria como al profesorado en activo.

Palabras clave: Primeros auxilios, reanimación cardiopulmonar, formación, profesorado, accidente escolar.
\end{abstract}

\begin{abstract}
In Spain, the educational legislation (LOMCE, 2013) includes the need to train students in First Aids and research indicates the importance of knowing Cardiopulmonary Resuscitation (CPR) techniques. In this context, with the aim of evaluating the knowledge of infant a primary school teacher $(\mathrm{N}=361)$ in $\mathrm{CPR}$, a descriptive study is carrying out using questionnaire divided into two parts, is applied: demographic data and questions related to theoretical knowledge in first aid. The results indicate that the teachers do not have knowledge in CPR and there are not significant differences with regard to age, gender, education and work experience ( $p>.05)$. There is a significant difference between those who have taken a course in first aid and those who have not. Based on the results, it is concluded that it is necessary to train in first aid, paying special attention to cardiopulmonary resuscitation, both for students in infant and primary education grades and for active teachers.
\end{abstract}

Keywords: First aid, cardiopulmonary resuscitation, formation, teachers, school accident.

\section{Introducción}

Los niños y las niñas constituyen el grupo social más vulnerable ante cualquier accidente. El informe de la Organización Mundial para la Salud, alerta de la gran carga de mortalidad y morbilidad que los accidentes y lesiones suponen (Peden et al., 2008), siendo estos la primera causa de muerte de los niños, niñas y adolescentes después del año en la mayoría de los países (Zayas, Cabrera \& Simón, 2007). Teniendo en cuenta que, en España, el alumnado de educación infantil y primaria (3-12 años) pasa un mínimo de 25 horas semanales en la escuela (LOMCE, 2013), no es de extrañar que la fundación Mafre (2014), en una investigación sobre los accidentes en la población infantil española, encuentre que el $57,2 \%$ de los accidentes que se producían fuera del hogar el 44,6\% ocurrían en la escuela. Estudios posteriores realizados en diferentes comunidades autónomas, también corroboran este dato: en Cataluña, las escuelas son el segundo espacio con mayor incidencia de accidentes (Brugulat, Medina \& Mompart, 2009); en la Comunidad Valenciana, el $31 \%$ de los accidentes en la población menor de 16 años tiene lugar en el entorno escolar (Viedma, Torner, Irles \& López, 2012) y; el servicio de salud de la Comunidad Autónoma del País Vasco, recoge que un 21,4\% de las lesiones no intencionadas acaecidas en menores de 14 años, se produce en centros escolares (Osakidetza, 2018). En el Estado español, las caídas, los traumatismos directos y las lesiones derivadas de actividades deportivas constituyen el $80 \%$ de los accidentes no intencionados (Arribas Bardón, Rivas, Mintegi \& Marañón, 2018). Si bien, tal y como recogen Smith y

Fecha recepción: 14-05-20. Fecha de aceptación: 15-07-20

PreferenciasZuriñe Gaintza

zuri.gaintza@ehu.eus
Colquhoun (2015), las paradas cardiorrespiratorias en niños y niñas son raras y una minoría de casos ocurren en la escuela, la supervivencia a una parada en edad pediátrica es baja (Rosell et al., 2016).

Ante esta realidad, saber realizar maniobras de reanimación cardiopulmonar (RCP) es de gran importancia para aumentar al máximo tanto las posibilidades de supervivencia como la calidad de vida de quien lo sufre (Strömsöe et al., 2015). Tal y como se recoge en la Guía de la European Resuscitation Council (Monsieurs et al., 2015), la inmediatez del inicio de la maniobra in situ y una actuación correcta, son factores que determinan o evitan tanto posteriores secuelas como incluso la mortalidad del individuo. Se sabe que, las posibilidades de supervivencia y de buena calidad de vida tras sufrirla son tiempo dependiente, de ahí la importancia de aplicar una RCP antes de la llegada de los servicios de emergencia (Koenraad et al., 2015; Weisfeldt et al., 2010) que, por lo general, tardan una media de 5 minutos o más en llegar al lugar del accidente (Fothergill et al., 2013). Una adecuada actuación dentro de los 3-5 minutos del colapso, aumenta las tasas de supervivencia entre un 50-70\% (Blom et al., 2014) siendo por lo general, personas capacitadas en RCP, no necesariamente del ámbito médico, las que realizan este tipo de actuaciones (Cave et al., 2011). De hecho, son numerosos los estudios que muestran como esta técnica es una actividad psicomotora que se puede aprender y desarrollar a partir de los 13 años (Fradejas \& Pérez, 2013). Organizaciones como la American Heart Association (2015) o la European Resuscitation Council (2015), señalan que la RCP realizada por testigos es crucial para la supervivencia en las paradas cardiacas extrahospitalarias. Si bien, cualquier persona que se encuentre cerca del sujeto que está en parada puede comenzar la reanimación, es más probable que personas que han recibido capacitación reciente ejecuten una maniobra 
correcta frente a aquellas que no recibieron dicha capacitación (Medtronic Foundation, 2013).

En esta dirección, el Consejo Español de Resucitación Cardiopulmonar (2015) insiste en la importancia de formar a todas las personas en el reconocimiento y actuación ante una parada cardiorrespiratoria (Perales \& Del Nogal, 2019). El hecho de que la educación sea obligatoria para todos los niños y niñas convierte al centro escolar en el lugar idóneo para conseguir la formación en RCP básica de toda la población (Isbye, Rasmussen, Ringsted \& Lippert, 2007). Pero, además, el hecho de que, en las escuelas, por lo general, no existan profesionales del ámbito sanitario (Navarrete, 2010), hace imprescindible la capacitación no sólo del alumnado (Ammirati, Gagnayre, Amsallem, Nemitz \& Gignon, 2014) sino que también, del profesorado (Sönmez, Uskun \& Pehlivan, 2014).

Así pues, parece primordial que ambos colectivos estén cualificados no sólo para el reconocimiento precoz de una parada, sino que, si fuera necesario, también para la realización de la propia maniobra de reanimación. Pero ¿están ambos colectivos realmente capacitados para reconocer y brindar los primeros auxilios ante una parada cardiorrespiratoria?

Por un lado, la investigación recoge que el profesorado, a nivel mundial, no tiene conocimientos en primeros auxilios (PPAA) en general y, menos aún, en RCP (Gaintza y Velasco, 2017), no siendo el Estado español una excepción (Abelairas et al., 2019; Alba, 2015). Por lo que, a la vista de los datos, la formación en RCP es necesaria (Amro \& Qtait, 2017; Navarro, Cons \& Romo, 2020).

Dada la alta probabilidad de que el accidente ocurra durante la sesión de educación física (Abernethy, MacAuley, McNally \& McCann, 2003; Longás, E., Longás, J. \& Riera, 2014), se ha investigado específicamente el conocimiento de los docentes de educación física en esta área. Si bien, Kumar et al. (2013) encuentran que estos profesionales puntúan significativamente mejor que el resto, ni el estudio de AlRobaiaay (2013), ni el de López, Navarro y Basanta (2015) encuentran estas diferencias. Desconocimiento que aumenta cuando se trata de aplicar RCP a infantes (Navarro et al., 2020). Por ello, desde el punto de vista médico, se recalca que el profesorado necesita una formación específica con las peculiaridades que conlleva la actuación en la edad pediátrica (Ammirati eta al., 2014; Lockey \& Georgiou, 2013), ya que, a esta edad, las causas más frecuentes que provocan o terminan en parada, son principalmente de origen respiratorio a diferencia de la de los adultos que son de origen cardiaco (Atkins et al., 2009). El profesorado, ante dicho requerimiento y conscientes de su desconocimiento, demanda una formación general en PPAA y en especial en RCP (Alonso \& Campo, 2014; Llorent \& Cobano, 2019).

Por otro lado, los niños y niñas, al ser un colectivo que obligatoriamente pasa por el sistema educativo, se convierten en grupo diana para conseguir una formación en RCP básica, reconociéndose la escuela como el lugar ideal para dicha instrucción (Miró, Díaz \& Sánchez, 2012). Con ello, a corto plazo, el alumnado está capacitado para poder salvar la vida de un compañero y, a largo plazo, se incrementa significativamente el número de adultos capacitados en RCP (Chamberlain \& Hazinski, 2003), constituye «per se» un co- nocimiento socialmente necesario. Por todo ello, la investigación concluye que esta instrucción debe comenzar como asignatura obligatoria en la escuela (Ammirati et al., 2014; De Buck et al., 2015) y actualizarse anualmente (Wilks \& Pendergast, 2017).

Si bien, esta propuesta puede parecer una idea reciente, la enseñanza de técnicas de RCP en las escuelas comenzó en Noruega en la década de los 60 (Lind \& Stovner, 1963) y, posteriormente, se introdujo en países como Escandinavia, Reino Unido y los Estados Unidos (Drezner, Rao, Heistand, Bloomingdale \& Harmon, 2009). En España, aunque la LOMCE (2013) incluye la necesidad de formar al alumnado en PPAA, ninguno de los decretos legislativos hace referencia explicita a la RCP. Con lo que, para las diferentes experiencias que en formación en RCP se realizan en las escuelas, es necesario conocer qué dice la investigación con relación a la misma para conocer la edad o características del escolar, el momento, la duración o los contenidos, etc. y así, realizar una formación eficaz y eficiente en el ámbito escolar. En concreto se sabe sobre:

Características de los escolares para ejecutar una respuesta apta ante una parada cardiorrespiratoria. Se ha visto que, a partir de la edad de 7 años los escolares son capaces de aprender, reproducir y retener los conocimientos teóricos en relación a la maniobra de RCP (Uray et al., 2003); que, a la edad de 9 años son capaces de poner en práctica (Ammirati et al., 2014); que, a la edad de 10 años son capaces de llevar a cabo una vigorosa compresión de pecho en un maniquí (Bohn, et al., 2012) y; que, a los 13 años pueden lograr una calidad de RCP similar a la de una persona adulta (Abelairas, Rodríguez, Casillas, Romo \& Barcala, 2014). No obstante, la correcta ejecución no va a depender sólo de la edad, sino que también, del peso del escolar (Jones et al., 2007).

Tiempo de la instrucción en RCP a escolares. Se considera que 3 sesiones anuales son suficientes para formar a los escolares en RCP (Colquhoun, 2012) si bien se ha visto que, con una sola sesión de una hora niños y niñas de 10 años adquieren los conocimientos teóricos mínimos requeridos (Bohn et al., 2012). Incluso la Organización Mundial de la Salud avala este planteamiento a través de la iniciativa «Kids save lives» que considera necesario incluir la RCP en el currículo escolar a partir de los 12 años con una carga formativa de no más de 2 sesiones al año (Bohn, Lukas, Breckwoldt, Böttiger \& Van Aken, 2015). No obstante, Reveruzzi, Buckley y Sheehan (2016) realizan una revisión bibliográfica sobre programas de formación y observan que, el tiempo de duración de estos varía según los objetivos del programa y las características del grupo de escolares.

El profesional apto para la instrucción en RCP a los escolares. Se ha visto que no existen diferencias entre la instrucción impartida por el profesorado o por instructores del ámbito médico (Plant \& Taylor, 2013). Tal y como recoge el estudio de Pichel et al. (2018), el profesorado, previamente entrenado, puede enseñar eficazmente los principios de RCP a su alumnado, (Campbell, 2012; Colquhoum, 2012; Lago, Basanta \& Navarro, 2018) siempre y cuando, el docente esté formado y conozca la realización de las maniobras cardiacas (Monsieurs et al. 2015; Sönmez et al., 2014).

Considerando la importancia que la investigación otorga 
al conocimiento del profesorado para la instrucción en RCP, el objetivo de este estudio es, evaluar el conocimiento del profesorado de educación infantil y primaria en RCP y detectar aquellas variables que puedan influir en dicho conocimiento: género, edad, antigüedad, etapa educativa, especialidad, titulación académica y formación en PPAA.

\section{Método}

\section{Diseño de investigación}

Este trabajo de investigación se encuadra dentro de los «estudios ex post facto» (Montero y León, 2005). En concreto, se presenta un estudio que, aunque no conlleva la manipulación o la intervención de ciertas variables, sí trata de determinar la relación entre variables dependientes (por ejemplo, el conocimiento del profesorado en RCP) con otras independientes, como haber participado en procesos formativos sobre dicha temática o pertenecer al cuerpo de profesorado de educación física entre otras.

Se presenta, por lo tanto, una investigación que responde a un diseño correlacional, descriptivo, de carácter cuantitativo y exploratorio, que se sitúa en el marco de una metodología por encuesta (Berends, 2006) propia de la investigación educativa (Tuckman \& Harper, 2012).

\section{Participantes}

La muestra estuvo compuesta de 361 maestros y maestras de 17 centros públicos de educación infantil y primaria de la provincia de Bizkaia (norte de España). En aplicación de la fórmula para el cálculo del tamaño muestral en poblaciones finitas, la muestra es representativa del total de los 8873 maestros y maestras que según Eustat (2017) había en dicha provincia en el curso escolar 2016-2017, año en el que se recogieron los datos. Este grado de representatividad de la muestra ha de considerarse para un nivel de confianza del $95 \%$ y un margen de error de \pm 5.05 . La diferencia entre géneros (hombres, $15.2 \%$ y mujeres $84.8 \%$ ) se explica por el porcentaje de maestros $(13.8 \%)$ y maestras $(86.3 \%)$ existentes en el colectivo total investigado. Las características de las y los participantes se recogen en la Tabla 1.

\begin{tabular}{|c|c|c|}
\hline Rasgos característicos & $\mathrm{N}$ & $\%$ \\
\hline \multicolumn{3}{|l|}{ Género } \\
\hline Mujer & 306 & 84.8 \\
\hline Hombre & 55 & 15.2 \\
\hline \multicolumn{3}{|l|}{ Edad (años) } \\
\hline$=33(24-33)$ & 41 & 11.4 \\
\hline $34-53$ & 245 & 67.8 \\
\hline$=54(54-64)$ & 75 & 20.8 \\
\hline \multicolumn{3}{|l|}{ Ciclo } \\
\hline Educación infantil & 127 & 35.2 \\
\hline Educación Primaria & 176 & 48.7 \\
\hline Ambos ciclos & 43 & 11.9 \\
\hline Dirección & 15 & 4.15 \\
\hline \multicolumn{3}{|l|}{ Ocupación } \\
\hline Tutor/a & 210 & 58.1 \\
\hline Específico & 114 & 31.5 \\
\hline Dirección & 15 & 4.15 \\
\hline Educación Física & 22 & 6.09 \\
\hline \multicolumn{3}{|l|}{ Estudios } \\
\hline Diplomatura & 346 & 94.4 \\
\hline Licenciatura & 82 & 22.7 \\
\hline Máster & 29 & 8.03 \\
\hline Experiencia (años) & & \\
\hline$=9(1-9)$ & $\begin{array}{c}51 \\
210\end{array}$ & $\begin{array}{l}58.0 \\
58.1\end{array}$ \\
\hline $10-29$ & 94 & 25.8 \\
\hline
\end{tabular}

\section{Instrumento}

En la fase inicial del estudio se desarrolló un cuestionario para medir el conocimiento en PPAA. Tras revisar diferentes investigaciones sobre PPAA, se optó por adaptar la escala de Abraldes y Ortín (2008) a la nueva normativa surgida desde el 2008 y los ítems se redactaron de una manera más explicativa. Los nuevos ítems generados fueron validados por un panel de tres expertos de la faculta de medicina de la Universidad del País Vasco, así como por formadores de la Cruz Roja. Con todo, el cuestionario tuvo dos bloques: el primero, con preguntas abiertas y cerradas recogía información de factores sociodemográficos para determinar las variables independientes relacionadas con el conocimiento: genero, edad, ciclo, estudios, ocupación (Tutor/a, Específico, Dirección y Educación Física), años de experiencia y formación en PPAA. Y el segundo, presentaba 27 preguntas cerradas sobre conocimientos generales en PPAA. En cuanto a su fiabilidad, el cuestionario obtuvo un alfa de Crombach de .967 para el total de los 27 items, y de .892 para las siete preguntas relacionadas a la RCP, lo que significa la obtención de unos valores que pueden ser categorizados como excelentes y buenos según George \& Mallery (2003). En concreto, en el campo de la RCP y se presentaron 7 preguntas de respuesta múltiple con una sola afirmación cierta:

Las ventilaciones (introducción de aire boca-boca o boca-nariz) en la resucitación cardiopulmonar básica $(R C P)$ se realizarán:

Respecto a la resucitación cardiopulmonar básica indica la opción más correcta:

Si nos encontramos con un alumno/a de 9 años, de $20 \mathrm{Kg}$. inconsciente en la pista y se le realiza la RCP, el ritmo de coordinación entre masaje cardiaco e insuflación (introducción de oxigeno en la vía aérea) será:

Sobre la insuflación del aire (introducción de aire en las vías aéreas) sabemos que:

Si un alumno/a permanece en el suelo tras una caída repentina, ¿Qué debemos hacer, si no responde a los estímulos, pero respira bien?:

Si un alumno/a permanece en el suelo tras una caída repentina, ¿Qué debemos hacer en primer lugar si no respira, esté inconsciente y tiene pulso?

Respecto a las compresiones torácicas para un alumno/a considerado adulto, la actuación correcta será:

Se otorgó «1» punto a las respuestas correctas y «0» a las incorrectas. Tras calcular las puntuaciones totales de cada participante, y siguiendo una estrategia similar a la utilizada por Abraldes y Ortín (2010), se categorizó su conocimiento, en base a la puntuación obtenida en RCP, atendiendo a cuatro rangos: $0-1$ punto, muy deficiente; $2-3$ puntos deficiente; 4-5-6 puntos, aprobado: y 7 puntos excelente.

\section{Procedimiento y análisis estadístico}

En un principio se accedió a la página web de la delegación de educación del Gobierno Vasco y se obtuvo una relación de todos los centros públicos de infantil y primaria de la provincia de Bizkaia. Se contactó con todos los centros educativos vía correo electrónico, adjuntando información sobre el proyecto, las características de la investigación e información sobre la participación referida al consentimiento informado, así como al anonimato de los participantes. En aquellos centros que estuvieron dispuestos a participar nos personalizamos con los cuestionarios impresos y se les dio 3 semanas para su cumplimentación, tras las cuales, nos per- 
sonalizamos en los centros para su recogida.

El análisis estadístico de los datos se realizó con el paquete estadístico SPSS (versión 23.0). Se generaron estadísticos descriptivos y análisis de frecuencia y porcentaje de las respuestas obtenidas. Se implementaron análisis de Chicuadrado para comparar las puntuaciones obtenidas atendiendo a las variables género, edad, ciclo, estudios, ocupación, años de experiencia y formación en PPAA. Con el fin de determinar si las diferencias en conocimiento observadas en el análisis descriptivo eran significativas, se realizó una comparación de medias paramétricas (t de Student). El nivel límite para determinar diferencias significas se estableció en $\mathrm{p}<$ 0.05

\section{Resultados}

Atendiendo a las variables demográficas, los participantes mayoritariamente fueron del género femenino y casi un $68 \%$ estuvo en la franja comprendida entre los 34 y 53 años. La formación de la mayoría fue de diplomatura $94.5 \%$ y un $58.2 \%$ tuvo una experiencia docente de entre 10 y 29 años.

En cuanto al conocimiento en RCP, tras el análisis de datos se observa que, por un lado, el $62.6 \%$ del profesorado tiene un conocimiento deficiente en materia de RCP, a los que se suma un $28.5 \%$ con conocimientos muy deficientes. Por otro lado, de los 361 participantes 29 (8\%) tienen un conocimiento apto en materia de RCP y 3 docentes $(0.8 \%)$ obtienen 7 puntos, la máxima puntuación (Tabla 2).

El análisis con respecto a diversas variables independientes nos dice que el nivel de conocimiento en RCP no está asociado a la edad, al género, a la etapa educativa ni a los años de experiencia como docente. En cuanto a la ocupación, considerando al grupo de profesionales en el área de educación física $(\mathrm{EF})$ frente al resto de profesionales, se realiza un análisis descriptivo (Tabla 3) de los dos grupos. La puntuación media de este profesorado (2.1818) es, ligeramente superior a la puntuación media del resto de los participantes (2.0708). La comparación de medias paramétricas entre ambos grupos (Tabla 3 ) muestra que no existen diferencias significativas $(\mathrm{p}=.634)$ entre ambos colectivos. De los 22 profesores/as de EF que rellenan el cuestionario, solo 2 superan el cuestionario en RCP.

Sin embargo, se observan diferencias significativas

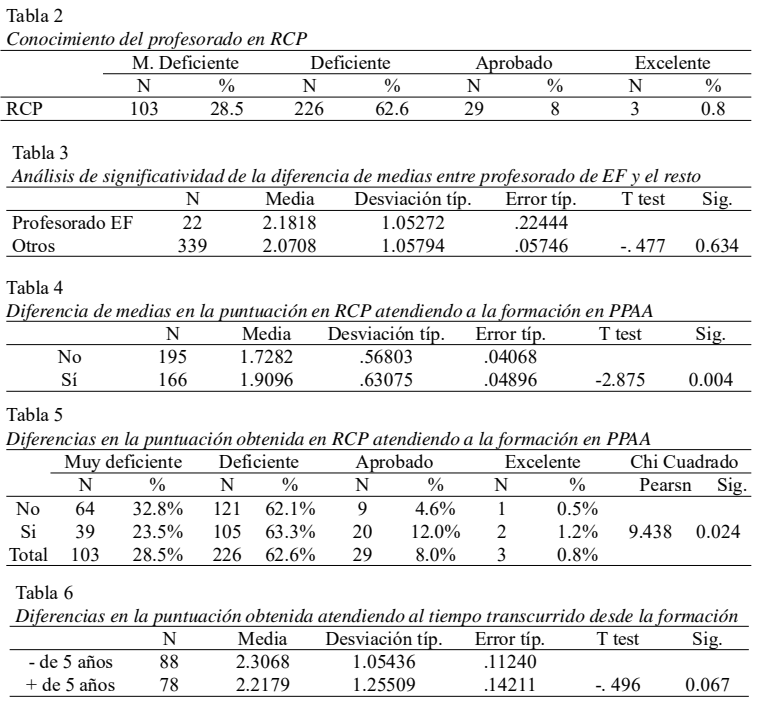

$(\mathrm{p}=.004)$ entre aquellas personas que han realizado un curso en PPAA y aquellas que no (Tabla 4). Concretamente, el grupo que ha recibido formación alcanza una media de 1.90, mientras que quienes no se han formado en PPAA logran una puntuación de 1.72 .

Si profundizamos en estos datos a partir de un estudio de la distribución (tabla 5) observamos que en ninguno de los grupos el 50\% de los participantes aprueba, aunque entre las 166 personas que ha recibido formación hay más sujetos que alcanzan el aprobado. Concretamente, la suma de aprobados y excelentes es un $13.2 \%$ frente al $5.1 \%$ de los que no reciben formación. Con ello también se deduce que, el $86.8 \%$ de los sujetos que tienen formación en PPAA no tienen conocimiento apropiado en RCP.

Tal y como se recoge en la tabla 5 , los resultados del profesorado que ha recibido formación en los últimos 5 años es ligeramente superior de la de aquellos que realizaron el curso hace más de 5 años.

Para determinar si estas diferencias observadas en el análisis descriptivo son significativas, se realiza la prueba $t$ de Student. Los resultados (Tabla 5) muestran que no existen diferencias significativas entre el tiempo transcurrido desde que se realizó la formación y el conocimiento en RCP $(p=.067)$.

Finalmente, para concretar el perfil de los 32 docentes que presentan un conocimiento apto en RCP se realiza un análisis con las variables demográficas. En el análisis se observa que, si bien la mayoría son mujeres $(84.4 \% \mathrm{M} \mathrm{y} 15.6 \% \mathrm{H})$ este porcentaje es casi el mismo que el que presenta la muestra total del estudio $(84.8 \% \mathrm{M}$ y $15.2 \% \mathrm{H})$. Lo mismo ocurre con la zona, el centro, la edad, la ocupación (tutor vs profesorado específico) y el ciclo en el que trabajan. El porcentaje de las personas que aprueban en RCP se distribuye en proporción a la muestra total.

\section{Discusión}

Sin ninguna duda, un paro cardiaco repentino en la escuela es potencialmente devastador para las familias y las comunidades (Rose et al., 2016). Debido al gran número de horas al día en las que el profesorado es responsable del bienestar físico de un gran número de niños y niñas, independientemente de la causa que lo provoque, es imprescindible que estos profesionales tengan un mínimo conocimiento en RCP (Lotfi et al., 2007). No obstante, el presente estudio observa que, el profesorado de educación infantil y educación primaria de la provincia de Bizkaia no tiene conocimiento suficiente para dar una respuesta adecuada y correcta para atender a un escolar en parada cardiorrespiratoria. Tan sólo un $8.86 \%$ de los participantes aprueba las preguntas en RCP. Tónica que se repite en todos los estudios que miden conocimiento en RCP del profesorado. Así, entre otros, en Holanda, Mpotos, Vekeman, Monsieurs, Deresee y Valcke (2013) encuentran que, si bien el 59\% de 2273 docentes había recibido formación previa en $\mathrm{RCP}$, el $61 \%$ de los mismos no se sentía preparado para impartir esta materia; en la India (Joseph et al., 2015), sólo un 5.47\%; en Arabia Saudi, un 11.9\% (Bashir \& Bakarman, 2014); en Eslovenia, encuentran que menos de un 20\% (Slabe \& Fink, 2013) y; en España, Navarro et al. (2020) encuentran que casi un 93\% de los 
participantes declaran no saber realizar una RCP en infantes. Este desconocimiento generalizado del profesorado a la hora de realizar las maniobras de RCP nos lleva a dos escenarios del contexto escolar en los que se precisa conocimiento y éste no existe.

Por un lado, está el contexto de ayuda a la persona en parada cardiorrespiratoria. Tal y como señalan Perkins et. al. (2015), se considera imprescindible que, si un escolar permanece en el suelo tras una caída repentina, el docente compruebe su estado de inconsciencia, llame al servicio de emergencia, abra la vía aérea, determine si respira o no, realice la respiración boca a boca, determine signos de vida y realice un masaje cardiaco. A la vista de los resultados de este estudio, compete, tanto a la delegación de educación del Gobierno Vasco ofertar cursos básicos en RCP al profesorado en activo como, a las universidades de la Comunidad Autónoma Vasca impartir en la formación inicial del profesorado contenidos referidos a esta formación básica en RCP. En este contexto de formación, es necesario recordar las directrices del Consejo Europeo de Reanimación que hace hincapié en enseñar al profesorado de infantil y primaria la RCP con las peculiaridades que conlleva la actuación en la edad pediátrica (Monsieurs et al., 2015). Además, conociendo que muchos de los accidentes ocurren en las clases de educación física, (Abraldes \& Ortín, 2008; Al-Robaiaay, 2013; Kumar et al. 2013, entre otros) la importancia de la formación del profesorado de educación física en esta área adquiere mayor interés. Contrariamente a lo esperado, el hecho de que en este estudio no existan diferencias significativas entre los profesionales de educación física y el resto se puede atribuir a dos razones: (1) la formación de diplomatura del 94,45 \% remite a los antiguos planes de estudio que no incluían formación en PPAA y a la vista de los resultados, cabe pensar que este colectivo, pese a su importancia, tampoco se ha formado posteriormente en esta materia y; (2) falta de asignatura específica en la titulación de magisterio. Quizá el profesorado de educación física más joven, pese a la indicación expresa del Libro Blanco (ANECA, 2004), no haya tenido ninguna asignatura en esta materia. Carencia que se recoge tanto, en la necesidad formativa que demanda el alumnado de magisterio en el estudio de González y Reche (2010) como en el estudio de Dopico (2016) en el que un 75\% de los participantes considera necesario incluir temas de PPAA en la formación del profesorado. De hecho, de las 61 universidades españolas que ofertan el grado de Maestra y Maestro en Educación Primaria tan sólo 3 tienen una asignatura específica en esta área (Navarro, Basanta, Abelairas \& López, 2015). No obstante, el hecho de que en el conocimiento en RCP en este estudio no existan diferencias significativas en ninguna de las variables estudiadas (género, edad, ocupación, formación y años de experiencia) se puede atribuir a, un descuido generalizado en la formación en esta materia de todo el profesorado en general, tal y como ya recoge Al-Robaiaay (2013).

Por otro lado, está el contexto de formación a los escolares. Tal y como señalan Wilks y Pendergast (2017), cualquier docente previamente formado puede impartir RCP de manera efectiva a su alumnado. Así, los participantes con formación en PPAA que puntúan mejor en RCP que los que no tienen dicha formación, se considerarían adecuados para impartir dicha formación. No obstante, la falta de conocimiento de- tectada, tan solo un $8.86 \%$ del profesorado supera la prueba, nos lleva a valorar la posibilidad de que, en un primer momento, profesionales del ámbito médico formen a los escolares en RCP. Por ejemplo, tal y como recogen Patsasi et al. (2012), estudiantes de medicina, profesionales de la salud, voluntarios de Cruz Roja, así como miembros de los servicios de emergencias pueden ser instructores alternativos.

\section{Conclusiones}

Este estudio demuestra que, independientemente de tener formación en PPAA o no tenerla, el profesorado de infantil y primaria no tiene conocimientos sobre la RCP, por lo que, por un lado, sin este conocimiento teórico, difícilmente podrá socorrer a un escolar en parada cardiaca o podrá instruir a su alumnado. De ahí que, las conclusiones de este estudio sean básicamente dos. Una, al igual que indican Abelairas, López, Martínez, Carballo y Rodríguez (2019), es necesario incluir en los grados de educación infantil y primaria formación en primeros auxilios poniendo especial atención en la reanimación cardiopulmonar, y dos, se precisa también de una inminente formación en RCP al profesorado en activo. Dado que el número de docentes que supera la prueba es muy bajo (sólo el 8.66\%), convendría llevar a cabo futuros análisis cuando la formación en PPAA sea más efectiva en el conocimiento de RCP.

\section{Referencias}

Abelairas, C, López, S., Martínez, S., Carballo, A., \& Rodríguez, A. (2019). Basic life support knowledge of the future of the Infant and Primary School teacher. An unresolved problem in university study plans? Annals Pediatría, 91(5), 344-345. doi: 10.1016/j.anpedi.2018.10.010

Abelairas, C., Carballo, A., Martínez, S., López, S., Rico, J., \& Rodríguez, A. (2019). Conocimientos y actitudes sobre los primeros auxilios y soporte vital básico de docentes de Educación Infantil y Primaria y los progenitores. Anales de Pediatría, 92(5), 268-276. doi: 10.1016/j.anpedi.2019.10.010

Abelairas, C., Rodríguez, A., Casillas, M., Romo, V., \& Barcala, R. (2014). Schoolchildren as life savers: At what age do they become strong enough? Resuscitation, 84(4), 814-819. doi: 10.1016/j.resuscitation.2014.03.001

Abernethy, L., MacAuley, D., McNally, O., \& McCann, S. (2003). Immediate Care of School Sport Injury. Injury Prevention, 9, 270-273. doi: 10.1136/ip.9.3.270.

Abraldes, J., \& Ortín, A. (2008). La Formación del Profesorado de Educación Física en PPAA. Almería, España: APEF

Abraldes, J., \& Ortín, A. (2010). Conocimiento en PPAA de los profesores de Educación Física en la ESO. Revista Internacional de Medicina y Ciencias de la Actividad Física y el Deporte, 10(38), 271-283.

Alba, R. (2015). Educación para la salud en PPAA dirigida al personal docente del ámbito escolar. Enfermería universitaria, 12(2), 88-92. doi: 10.1016/j.reu.2015.04.004

Alonso, N., \& Campo, M. (2014). Necesidad percibida de formación sanitaria de los profesores de Primaria. Nuberos Científica, 2(13), 25-31

Al-Robaiaay, Y. (2013). Knowledge of Primary School Teachers Regarding First Aid in Baghdad, Al-Kindy College. Medical Journal, 9, 54-59. 
American Heart Association (2015). Guidelines CPR \& ECC. https://www. eccguidelines.heart.org/index.php/americanheart-association/ Acceso junio, 2019

Ammirati, C., Gagnayre, R., Amsallem, C., Némitz, B., \& Gignon, M. (2014). Are Schoolteachers Able to Teach First Aid to Children Younger than 6 Years? A comparative study. BMJ open, 4(9), 27-48. doi:10.1136/bmjopen-2014-005848

Amro, N., \& Qtait, M. (2017). General Knowledge \& Attitude of First Aid among Schoolteacher's in Palestine. International Journal of Innovative Research in Medical Science, 2(4), 660-665. doi: 10.23958/ijirms/vol02-i4/05

ANECA, (2004). Libro Blanco. Título de Grado en Magisterio, vol. I y II. Madrid.http://www.aneca.es/var/media/150404/ libroblanco_jun05_magisterio1.pdf. Acceso Junio, 2019.

Arribas, C., Bardón, E. J., Rivas, A., Mintegi, S., \& Marañón, R. (2018). Consultas relacionadas con lesiones no intencionadas en urgencias en España: serie de casos. Anales de Pediatría, 89(6), 333-343.

Atkins, D., Everson, S., Sears, G., Daya, M. Osmond, M. Warden, C., \& Berg, R. (2009). Epidemiology and outcomes from outof-hospital cardiac arrest in children: The Resuscitation Outcomes Consortium Epistry-Cardiac Arrest. Circulation, 119, 1484-1491. doi: 10.1161/CIRCULATIONAHA.108.802678

Bashir, S., \& Bakarman, M. (2014). Are our children in safe hands? Evaluating the preparedness of primary school staff in Jeddah, Saudi Arabia in responding to health-related emergencies. Life Science Journal, 11(11), 986-989.

Berends, M. (2006). Survey Methods in Educational Research. In J. L. Green, G. Camilli, \& P. B. Elmore (Eds.), Handbook of complementary methods in education research (p. 623-640). Lawrence Erlbaum Associates Publishers.

Blom, M., Beesems, S., Homma, P., Zijlstra, J., Hulleman, M., van Hoeijen, D... Koster, R. (2014). Improved survival after outof-hospital cardiac arrest and use of automated external defibrillators. Circulation, 130(21), 1868-1875. doi:10.1161/ circulationaha.114.010905

Bohn, A., Van Aken, H., Möllhoff, T., Wienzek, H., Kimmeyer, P., Wild, E., ... Weber, T. (2012). Teaching resuscitation in schools: annual tuition by trained teachers is effective starting at age 10. A four-year prospective cohort study. Resuscitation, 83, 619-625. doi:10.1016/j.resuscitation.2012.01.020

Bohn, A., Lukas, R., Breckwoldt, J., Böttiger, B., \& Van Aken, H. (2015). "Kids save lives": Why schoolchildren should train in cardiopulmonary resuscitation. Curren Opinion Critical Care, 21,220-5. doi: 10.1097/MCC.0000000000000204

Brugulat, P., Medina, A., \& Mompart, A., (2009). La Salut de la Població Infantil a Catalunya: Enquesta de Salut de Catalunya 2006. $1^{a}$ ed. Catalunya: Direcció General de Planificació i Avaluació.

Campbell, S. (2012). Supporting Mandatory First Aid Training in Primary Schools. Nursing Estándar, 27(6), 35-39.

Cave, D., Aufderheide, T., Beeson, J., Ellison, A., Gregory, M., Hazinski, ... Schexnayder, S. (2011). Importance and implementation of training in cardiopulmonary resuscitation and automated external defibrillation in schools: A science advisory from the American Heart Association. Circulation, 123, 691-706. doi:10.1161/CIR.0b013e31820b5328

Chamberlain, D., \& Hazinski, M. (2003). Education in resuscitation: an ILCOR symposium. Circulation, 108, 25752594. doi: 10.1161/01.CIR.0000099898.11954.3B

Colquhoun, M. (2012). Learning CPR at school-Everyone should do it. Resuscitation, 83, 543-544.

Consejo Español de Resucitación Cardiopulmonar (2015). De- claración del consejo español de resucitación cardiopulmonar sobre las nuevas recomendaciones del consejo europeo de resucitación. www.erc.edu

De Buck, E., Van Remoortel, H., Dieltjens, T., Verstraeten, H., Clarysse, M., Moens, O., \& Vandekerckhove, P. (2015). Evidence-based Educational Pathway for the Integration of First Aid Training in School Curricula. Resuscitation, 94, 822. doi: 10.1016/j.resuscitation.2015.06.008

Dopico, M. (2016). Estudio descriptivo sobre la formación inicial de los/as maestros y maestras de EF escolar. Sportis, 2(2), 188-205. doi: 10.17979/sportis.2016.2.2.1429

Drezner, J., Rao, A., Heistand, J., Bloomingdale, M., \& Harmon, K. (2009). Effectiveness of emergency response planning for sudden cardiac arrest in United States high schools with automated external defibrillators. Circulation, 120, 518-25. doi: 10.1161/CIRCULATIONAHA.109.855890

Eustat, (2017). Eskola-jardueraren estatistika 2016/2017 ikasturtea, https://goo.gl/j7vUgP. Acceso, julio, 2020.

Fothergill, R., Watson, L., Chamberlain, D., Virdi, G., Moore, F., \& Whitbread, M. (2013). Increases in Survival from Out-OfHospital Cardiac Arrest: A Five-Year Study. Resuscitation, 84(8), 1089-1092. doi: 10.1016/j.resuscitation.2013.03.034.

Fradejas, V., \& Pérez, P. (2013). Importancia de una comunidad educativa formada en técnicas de RCP, Nuberos Científica, 3(10), 13-17.

Gaintza, Z., \& Velasco, Z. (2017). Análisis del grado en formación en PPAA del profesorado en activo de infantil y primaria. Formación Universitaria, 10(2), 67-78. doi: 10.4067/S071850062017000200008

George, D., \& Mallery, P. (2003). SPSS for Windows step by step: A simple guide and reference 11.0 update ( $4^{\text {th }}$ ed.). Boston: Allyn \& Bacon

González, I., \& Reche, E. (2010). Las Demandas Formativas del Alumnado de Magisterio. Construcción de un Plan de Formación Complementaria. Magis: Revista Internacional de Investigación en Educación, 2(4), 383-400.

Isbye, D.L., Rasmussen, L.S., Ringsted, C., \& Lippert, F.K. (2007). Diseminating CPR training by distributing 35,000 personal manikins among school children. Circulation, 116, 13801385.

Jones, I., Whitfield, R., Colquhoun, M., Chamberlain, D., Vetter, N., \& Newcombe, R. (2007). At what age can schoolchildren provide effective chest compressions? BMJ, 334, 1201-15. doi:10.1136/bmj.39167.459028.DE

Joseph, N., Narayanan, T., bin Zakaria, S., Venugopal Nair, A., Belayutham, L., \& Subrmanian, A. M. (2015). Awareness, Attitudes and Practices of First Aid Among School Teachers in Mangalore. Journal of Primary Health Care, 7(4), 274281. doi: $10.1071 / \mathrm{HC} 15274$

Koenraad G. Monsieurs, J. P. Nolan, L. L... Bossaert, T. (2015). European Resuscitation Council Guidelines for Resucitation. Section 1. Executive summary. Resuscitation, 95, 1-80. doi: 10.1016/j.resuscitation.2015.07.038.

Monsieurs, K.G., Nolan, J.P., Bossaert, L.L., Greif, R., Maconochie, I.K., Nikolaou, N.I. ... Zideman, D.A. (2015). European Resuscitation Council Guidelines for Resucitation 2015 Section 1. Executive summary. Resuscitation, 95, 1-80. doi: 10.1016/j.resuscitation.2015.07.038

Kumar, S., Kulkarni, P., Srinivas, N., Prakash, B., Hugara, S., \& Ashok, N. (2013). Perception and Practices Regarding First Aid Among School Teachers in Mysore. National Journal of Community Medicine, 4, 349-52.

Lago, J., Basanta, S., \& Navarro, R. (2018). La enseñanza de 
PPAA en educación física: revisión sistemática acerca de los materiales para su implementación. Retos, Nuevas tendencias en Educación Física, deporte y recreación, 34, 349355.

Lind, B., \& Stovner, J. (1963). Mouth to mouth resuscitation in Norway. Journal of the American Medical Association, 185, 933-935.

Lockey, A., \& Georgiou, M. (2013). Children Can Save Lives. Resuscitation, 84(4), 399-400. doi: 10.1016/ j.resuscitation.2013.01.011

Lotfi, K., White, L., Rea T., Cobb, L., Copass, M., Yin, L... Eisenberg, M. (2007). Cardiac arrest in schools. Circulation, 116,13749. doi: 10.1161/CIRCULATIONAHA.107.698282

LOMCE (2013). Ley Orgánica 8/2013, de 9 de diciembre, para la Mejora de la Calidad Educativa. España: Boletín Oficial del Estado.

Longás, E., Longás, J., \& Riera, J. (2014). Daños No Intencionados en la Escuela. Estudio en Escolares de Cataluña. Educar, 50(2), 243-263. doi: 10.5565/rev/educar.42

López, R., Navarro, R., \& Basanta, S. (2015). Formación y actitud del profesorado de EF en educación Primaria con respecto a los PPAA en Lugo. Trances, 7(1), 91-112.

Mafre. (2014). Accidentes de la Población Infantil Española. https://www.fundacionmapfre.org/documentacion/publico/ i $18 \mathrm{n} / \mathrm{c}$ a t a $10 \mathrm{~g} \mathrm{o}$ i m a g e n e s / imagen.cmd?path=1078066\&posicion=1. Acceso Septiembre, 2019.

Medtronic Foundation. (2013). Every Second Counts. Every Action Matters, A Community Response Planning Guide for Sudden Cardiac Arrest. HeartRescue Project. http:// www.heartrescueproject.com/wcm/groups/mdtcom_sg/ @mdt/@corp/@fndn/@heartrescue/documents/images/ hrp-brochure.pdf. Acceso Mayo, 2019.

Miró, O., Díaz, N., \& Sánchez, M. (2012). Aprender reanimación cardiopulmonar desde la escuela. Emergencias, 24, 423-5.

Montero, I., \& León, O. G. (2005). Sistema de clasificación del método en los informes de investigación en Psicología. International Journal of clinical and health psychology, 5(1), 115-127.

Mpotos, N., Vekeman, E., Monsieurs, K., Derese, A., \& Valcke, M. (2013). Knowledge and Willingness to Teach CPR: A Survey Amongst 4273 Teachers. Resuscitation, 84(4), 496500. doi: 10.1016/j.resuscitation.2013.01.023

Navarrete R. (2010). La Actuación del Docente en EF ante el Accidente Escolar. Revista Portaldeportivo, 19, 4-15.

Navarro, R., Basanta, S., Abelairas, C., \& López, S. (2015). Análisis de la situación de los PPAA en los planes de estudio de los grados de maestra/o de Primaria. Trances, 7(4), 599-612.

Navarro, R., Cons, M., \& Romo, V. (2020). Conocimientos en soporte vital básico del profesorado gallego de educación infantil, primaria y secundaria: estudio transversal. Retos, Nuevas tendencias en Educación Física, deporte y recreación, 38, 172-178.

Osakidetza, (2018). Encuesta de Salud del País Vasco. Administración de la CAPV. Departamento de Salud, 1-83. https:// www.euskadi.eus/informacion-encuesta-salud-2018-tablasde-resultados/web01-a3osag17/es/Acceso, julio, 2020.

Peden, M., Oyegbite, K., Ozanne, J., Hyder, A.A., Branche, C., FazlurRahman,A.K.M., Rivara, F., \& Bartolomeos, K. (2008). World Report on Child Injury Prevention. Geneva: World Health Organization and UNICEF

Perales, N., \& Del Nogal, F. (2019). Una estrategia para el Sistema Nacional de Salud ante la parada cardiaca (1st ed., pp. 35-52). Valladolid: MATA Digital, S.L

Perkins, G, Lall, R., Quinn, T., Deakin C., Cooke M., Horton, J., \& Smyth, M. (2015). Mechanical Versus Manual Chest Compression for Out-Of-Hospital Cardiac Arrest (PARAMEDIC). Lancet, 385(9972), 947-955. doi: 10.1016/ S0140-6736(14)61886-9.

Pichel, M., Martínez, S., Barcala, R., Fernández, F., Vázquez, D., Sánchez, L., \& Rodríguez, A. (2018). Un primer paso en la enseñanza del soporte vital básico en las escuelas: la formación del profesorado. Anales de Pediatría, 89(5), 265-271. https://doi.org/10.1016/j.anpedi.2017.11.002

Plant, N., \& Taylor, K. (2013). How best to teach CPR to schoolchildren: a systematic review. Resuscitation, 84, 415421. doi: h10.1016/j.resuscitation.2012.12.008

Reveruzzi, B., Buckley, L., \& Sheehan, M. (2016). School-Based First Aid Training Programs: A Systematic Review. Journal of School Health, 86(4), 266-272. doi:10.1111/josh.12373

Rose, K., Martin, M., Berger, S., Courson, R., Fosse, G., Gillary, R., ... Thompson, A. (2016). Cardiac Emergency Response Planning for Schools. NASN School Nurse, 31, 263-70. doi: 10.1177/1942602X16655839

Rosell, F., Mellado, F. J., López, J. B., Fernández, P., Ruiz, M. M., González, I., ... García del águila, J. (2016). Supervivencia y estado neurológico de la parada cardiaca extrahospitalaria en edad pediátrica en Andalucía. Medicina Intensiva, 40(3), 163-168. doi: 10.1016/j.medin.2015.05.008

Smith, C. M., \& Colquhoun, M. C. (2015). Out-of-hospital cardiac arrest in schools: A systematic review. Resuscitation, 96, 296-302. doi: 10.1016/j.resuscitation.2015.08.021

Slabe, D., \& Fink, R. (2012). Kindergarten teachers and their assistant's knowledge of first aid in Slovenian. Health Education J., 72, 398-407. doi: 10.1177/0017896912446555

Sönmez, Y., Uskun, E., \& Pehlivan, A. (2014). Knowledge Levels of Pre-School Teachers Related with Basic First-Aid Practices, Isparta Sample. Turkish Archives of Pediatrics, 49, 238-246. doi:10.5152/tpa.2014.1581

Strömsöe, A., Svensson, L., Axelsson, A., Claesson., A., Göransson, K., Nordberg, P., \& Herlitz, J. (2015). Improved outcome in Sweden after out-of-hospital cardiac arrest and possible association with improvements in every link in the chain of survival. European Heart J., 36(14), 863-871. doi:10.1093/eurheartj/ehu240

Tuckman, B. W., \& Harper, B. E. (2012). Conducting educational research. Rowman \& Littlefield Publishers.

Uray, T., Lunzer, A., Ochsenhofer, A., Thanikkel, L., Zingerle, R.... Lillie, P., (2003). Feasibility of life-supporting first aid (LSFA) training as a mandatory subject in primary schools. Resuscitation, 59(2), 211-220. doi: 10.1016/S03009572(03)00233-8

Viedma, P., Torner, M., Irles, M., \& López, R. (2012). Encuesta de Salud de la Comunitat Valenciana. $1^{\text {a }}$ ed. Generalitat Valenciana: Consejería de sanitat. http:/www.san.gva.es/ documents/153218/167513/encuesta2010completo.pdf; ISBN: 978-84-482-5746-0. Acceso, octubre, 2019.

Wilks, J., \& Pendergast, D. (2017). Skills for life: First aid and CPR in schools. Health Education Journal, 76(8), 1009-1023. doi:10.1177/0017896917728096

Zayas, R., Cabrera, U., \& Simón, D. (2007). ¿Accidentes infantiles o lesiones no intencionales? Revista Cubana de Pediatría, 79(1), 1-15. 